\title{
Radiant Source Signal Recognition Algorithm based on SAE
}

\author{
Ye Wen-qiang, Yu Zhi-fu, Zhang Kui, and Wang Hu-bang \\ National University of Defense Technology, hefei, China
}

\begin{abstract}
Aiming at the shortcomings of traditional radar identification based on artificial judgment and module matching, this paper proposes an intelligent identification algorithm based on joint time-frequency. The radar radiation source signal is transformed by time-frequency, and the processed signal is input into the automatic encoder through different kinds of dimensionality reduction methods, and the pre-training adjustment depth learning model is adopted, and the commonly used softmax classifier is adopted to the pre-training model. Oversee fine school and identification, and finally complete the identification task. The simulation results show that high recognition rate can be achieved by this algorithm, and the joint dimension reduction is better than other methods.
\end{abstract}

\section{Introduction}

Radar emitter signal recognition plays an important role in the modern war, directly affects the performance of electronic reconnaissance equipment and related to the subsequent operational decision, it is not only the purpose of signal processing of the reconnaissance system, is an important basis for determining the enemy threat situation, plays an important role in radar electronic warfare in the process. Modern information warfare has put forward higher requirements for automatic and intelligent radar emitter recognition. The traditional method of emitter recognition based on artificial decision and template matching has been unable to adapt to the rapidly changing battlefield environment. Therefore, we need to further study artificial intelligence technology and realize signal automatic identification [1]. Radar emitter signal feature extraction, as the basis of signal sorting and recognition, directly determines the efficiency and accuracy of signal recognition, and then affects the capability of electronic intelligence reconnaissance and related to subsequent operational decision making [2].

In recent years, deep learning has attracted the attention of many scholars. In November 2012, a Microsoft event in Tianjin on Chinese public demonstration of a fully automatic simultaneous translation system, the speaker with English speech, the background of the computer automatically coherent speech recognition, speech synthesis and the Machine Translation Chinese, the effect is very smooth. In 2012, Hinton used convolution neural network to achieve remarkable achievements in the ImageNet competition. In view of the performance of the deep learning structure, this paper proposes the use of deep learning to identify the radar emitter signal [3].

\section{Step and Method of Signal Recognition}

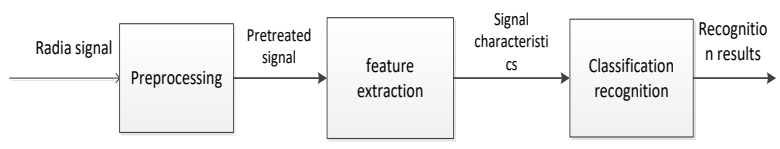

Fig. 1. Signal recognition flow chart

It can be concluded from the above signal recognition process in figure [4], first of all to the radar emitter signal preprocessing, this paper is mainly extracted by short time Fourier transform, and then by using SVD (locally linear embedding), LDA (linear discriminant analysis) for dimensionality reduction, data reduction to get into depth study of structure for classification and recognition.

\subsection{Feature Selection Based on Short Time Fourier Transform}

In 1946 Gabor proposed short-term Fu Liye transform (Short Time Fourier Transform, STFT), the basic idea is to take on a limited time window function in signal STFT, realize the localization signal in time domain, assuming the time window signal is stationary, moving in time axis on the signal segment of Fourier

\footnotetext{
* Corresponding author:869440437@qq.com
} 
transformation through the window, so as to obtain the time-varying characteristics of signals[5]. The STFT of the signal is defined as follows

$$
S(t, f)=\int_{-\infty}^{+\infty} S(\tau) h(\tau-t) e^{-j 2 \pi f \tau} d \tau
$$

The $\mathrm{H}(\mathrm{T})$ is the window function, moving along the time axis, obviously, if you take an infinite window function $\mathrm{H}(\mathrm{T})=1, \mathrm{t}$, STFT degradation for the traditional Fourier transform. The signal $\mathrm{s}(\mathrm{T})$ multiplied by a fairly short window function is equal to the slice taken from the signal at the time point $\mathrm{t}$, so STFT can be understood as the Fourier transform (local spectrum) near the $\mathrm{T}$ of "analysis time".

\subsection{Deep Learning Structure}

The automatic encoder is a typical three layer neural network structure proposed by Rumelhart in 1986. It consists of the input layer, the hidden layer and the output layer. The dimension of the input layer and the output layer are equal, and the dimension of the hidden layer is $\mathrm{m}$ dimension[6].

The encoder is formed between the input layer and the hidden layer, the input signal $x \in R^{n}$ containing $a \in R^{m}$ excitation characteristics of the data in the hidden layer through the encoding process between the hidden layer and the output layer are y decoder, signal reconstruction in $R^{n}$ through the decoding process. Since the training goal is to make the original encoder input is $y \approx x$, so as to obtain the most important data information in [7]. The process of the self encoder is expressed as a formula:

$$
\begin{aligned}
& \mathrm{a}=f\left(W_{a} X+b_{a}\right) \\
& \mathrm{b}=f\left(W_{v} a+b_{v}\right)
\end{aligned}
$$

Formula (2)and (3) $\mathrm{W}_{a} \in R^{m \times n}$ and $\mathrm{W}_{y} \in R^{d \times n}$ respectively, the weight matrix encoding and decoding layer layer, and meet the $W_{a}=W_{y}^{T}, b_{x} \in R^{m}$ and $b_{y} \in R^{d}$ respectively, encoding and decoding layer layer $f()$ for the bias term. The activation function, usually use the sigmoid function or tanh function. The sigmoid function is $f(z)=\frac{1}{1+e^{-z}}$, the range is $[0,1]$, the tanh function $f(z)=\frac{e^{z}-e^{-z}}{e^{z}+e^{-z}}$. The output data of $\mathrm{Y}$ output layer can be regarded as the input data of $\mathrm{X}$ input layer prediction, parameter auto encoder can use BP neural network algorithm to adjust the transmission, when the proximity of input data of $\mathrm{X}$ output layer of $\mathrm{Y}$ output data and the input layer is acceptable, then the auto encoder has retained most of the information of the original input the automatic data, the neural network trained encoder [8]. Definition of cost function

$$
J(W, b)=\left[\frac{1}{m} \sum_{i=1}^{m}\left(\frac{1}{2}\left\|y_{i}-x_{i}\right\|^{2}\right)\right]+\frac{\lambda}{2} \sum_{l=1}^{n_{1}-1} \sum_{i=1}^{s_{l}} \sum_{j=1}^{s_{i}+1}\left(\mathrm{~W}_{j i}^{(I)}\right)^{2}
$$

In formula $4, x_{i}$ and $y_{i}$ denote the input and output of the 1 samples of the encoder respectively. $W_{i j}^{(1)}$ represents the weights of neurons in the L level of the neuron, $\mathrm{B}$ is the bias item, $\mathrm{M}$ is the number of nodes, $n_{l}$ represents the number of network layers, and $s_{l}$ represents the number of neurons in the L level. The second term of the formula is the weight attenuation term of the weight attenuation coefficient [lambda], and the weight attenuation term plays the role of reducing the weight and preventing over fitting[9], The best parameter(W,b)is sought from the encoder, making the cost function $J(\mathrm{~W}, \mathrm{~b})$ smallest. Then it is considered that the output $y$ of the self encoder reconstructs the input $\mathrm{x}$, and the output a of the hidden layer is the characteristic expression of the input X.

\subsection{Softmax Classifier}

The encoder itself does not have the automatic classification function, the classification accuracy rate to determine the learning performance of auto encoder, so behind the auto encoder model adds depth classification layer, then the gradient descent method of parameter of network training[10], can be used for classification tasks, common classifiers are mainly Softmax classifier, SVM classifier, Bias classifier[11]. In this paper, softmax classifier is selected. The Softmax classifier can be used to solve the multi classification problem, that is, the number of desirable values of the sample label $\mathrm{y} \mathrm{k}$ satisfies the $\mathrm{k}>2$. Now a training set $\left\{\left(x^{(1)}, y^{(1)}\right), \cdots\left(x^{(m)}, y^{(m)}\right)\right\}$, in which the $y^{(i)} \in\{1,2 \cdots k\}$. This model is a supervised learning algorithm. The categories of the input samples are very clear, and the same sample can not belong to multiple categories at the same time. Assuming that the input is $\mathrm{x}$, then it is the probability of the class $J$ as $p(y=j / k)$ [12]. Specifically, the hypothesis function of the neural network corresponding to the classifier is 


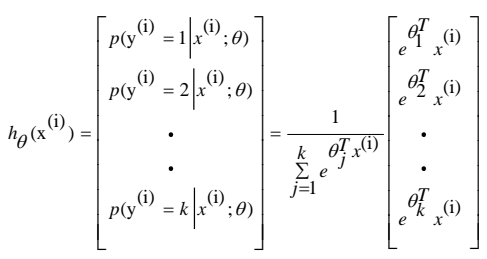

\subsection{Algorithm Framework for This Paper}

Step 1:the short-time Fourier transform of six kinds of emitter signals is used to reduce the dimension of the time-frequency images, and SVD and LDA dimensions are reduced respectively, and the high-dimensional input samples are formed.

Step 2: the high dimensional eigenvector is used as the input of the sparse self encoder, and the layer by layer greedy training method is used to carry out unsupervised pre training for the samples. A sparse self encoder is used to learn the deep features of the input features.

Step 3: after the completion of pre training, fine School of pre trained model using labeled training samples, this paper uses softmax classifier to get the features of SAE are classified according to the classification results and data label distance of network weights in tune with back propagation algorithm. So as to achieve the effect of fine tune, and get a good training network.

Step 4: the data of the test samples are sent to the trained network for classification and recognition, so as to realize the recognition of the signal.

\section{Simulation Analysis}

The data used in this article to the radar emitter signal MATLAB simulation, respectively conventional pulse signal $(\mathrm{CW})$, linear frequency modulation signal (LFM), nonlinear frequency modulation signal (NLFM), phase encoding signal compression (BPSK), two (BFSK) encoding signal frequency and the four frequency encoding signal (QFSK). The carrier frequency of CW, LFM, NLFM, BPSK and QPSK is set to $200 \mathrm{MHz}$, LFM frequency offset is set to $50 \mathrm{MHz}$, BPSK uses 13 bit Barker code, QPSK uses 16 bit Barker code, BFSK's two carrier frequency is QPSK, and 13 bit Barker code, four carrier frequency is $100 \mathrm{MHz}$, $300 \mathrm{MHz}, 500 \mathrm{MHz}, 700 \mathrm{MHz}$.Each signal has 600 samples under the $-4 \mathrm{~dB}$ to $4 \mathrm{~dB}$ signal to noise ratio, of which 500 are used as training networks and 100 are used for testing. The test hardware platform is CPUi57300 , the memory is $8 \mathrm{G}$, and the MATLAB version is R2011a.

After dimensionality reduction, this paper selects the self encoders that include two hidden layers. The original input feature in this paper is 625 dimensional, the output type is 6 , the number of hidden layer neurons in order to select the ideal, only need to change the number of the neurons in the first layer and the second layer, will bring the same data to the same network parameter, here the dimension reduction by SVD reduction to adjust network dimensional model. And the softmax classifier commonly used on the pre training end model adopts supervised sizing and recognition, to get the first layer of 151 neurons, second layer 100 best. The node structure of the neural network is 625-151-100-6.

Table. 1 Emitter signal identification accuracy with different dimensionality reduction

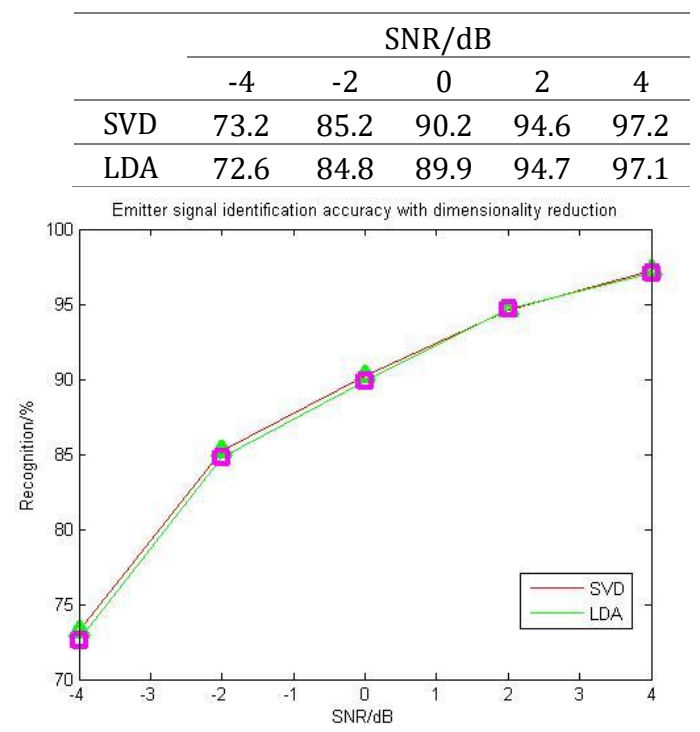

Fig.2. Signal recognition rate contrast curve of two dimensionality reduction methods

The simulation results are shown in Table 1. From the graph, we can see that with the increase of SNR, the recognition rate of radar emitter signals is increasing. After $\mathrm{SNR}=4 \mathrm{~dB}$, the recognition rate of these two algorithms is basically the same, and can reach more than $97 \%$.

The same data is combined to reduce the dimension through SVD and LDA, and then the data is entered into the same depth model structure as the simulation above, and the result of Table 2 is obtained.

Table. 2 Emitter signal identification accuracy with joint dimensionality reduction

\begin{tabular}{cccccc}
\hline & \multicolumn{5}{c}{$\mathrm{SNR} / \mathrm{dB}$} \\
\cline { 2 - 6 } & -4 & -2 & 0 & 2 & 4 \\
\hline SVD+LDA & 78.6 & 86.7 & 93.6 & 96.5 & 98.8 \\
\hline
\end{tabular}




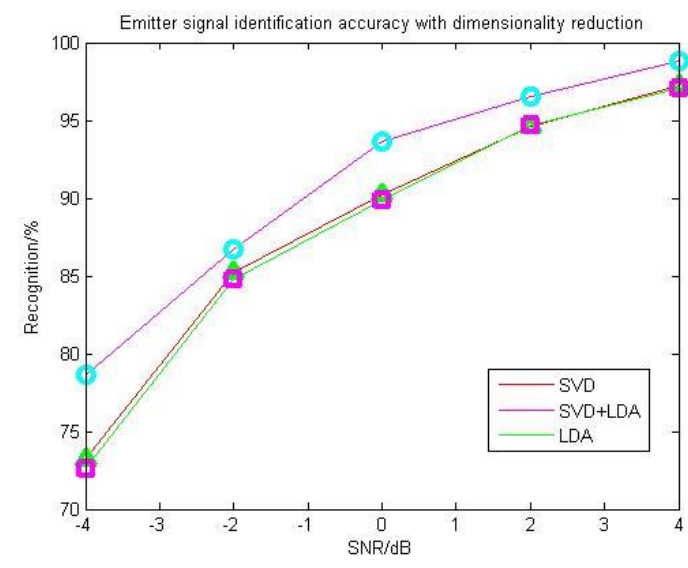

Fig.3.Signal recognition rate contrast curve of three dimensionality reduction methods

Through the simulation above, it is clear that the recognition rate through joint dimension reduction is obviously better than any one of the reduction methods only. At the time of $\mathrm{SNR}=4 \mathrm{~dB}$, the recognition rate has reached over $98 \%$. Through the combination of dimension reduction can improve the recognition rate of the reasons is the result of SVD reduction can contains the kernel of the principal component analysis (PCA), LDA can make small and large variance within class variance between classes, the two dimension reduction based on the advantage, reduce the dimensionality of the data features to keep better, which can improve the recognition rate.

\section{Summary}

This paper in the data preprocessing stage, in order to reduce the amount of calculation, the signal after short time Fourier transform for dimensionality reduction, using SVD, LDA and SVD+LDA three kinds of dimensionality reduction methods, and compared the simulation, the simulation results show that after the combined reduction can keep the data with more features, more conducive to classification. The recognition effect is better.

\section{References}

1. Bai Hang. Radar Emitter Signal Recognition Based on Time Frequency Analysis[D]. Journal of The PLA Information Engineering University.(2012).

2. Waltz E. The principle and practice of information warfare [M].(2004).

3. Deng Liu,Wang Zi-jie. Research on vehicle recognition based on deep convolution neural network[J]. Application Research of Computers. 2015,33(9),17-23.

4. Zhang Qin, Liu Yian.A new method of radar signal recognition[J]. Microcomputer \& Its Applications. 2015,34(12):48-53.

5. Guan Hui. Study Of Fault Diagnosis of Rolling Bearing Based On the Wavelet Analysis [D]. Taiyuan University of science \& Technology. (2008).
6. Deng Jun-feng,Zhang Xiao-long.Deep learning algorithm optimization based on combination of auto-encoders[J].Journal of Computer Applications . 2016,36(3):697-702.

7. Jose Dolz Nacim Betrouni, el al. Stacking denosing auto-encoder in a deep network to segment the brainstem on MRI in brain cancer patients: A clinical study[J].Computerized Medical Imaging and Fraphics,2016, $52: 8-18$.

8. Li Fei Gao Xiao-Guang Wan Kai-Fang . Reasearch on RBM Networks Training Based on Improvd ParaSVD Tempering Algorithm[J]. Acta-Automatica Sinica.2017,43(5):753-764.

9. Baldi P Guyon G Dror V, et al. Autoencoders Unsupervised Learning,and Deep Architectures Editor[J].Journal of Machine Learning Research, (2012).

10. XIE H, WANG S, LIU K, et al. Multilayer feature learning for polarimetric synthetic radar data classification[J]. IEEE Geoscience and Remote Sensing Symposium, 2014, 2818-282.

11. Chen Jin-lian,Huang Hou-kuan Tian Feng-zhan Qiu Tao-rong.Effective Classifier for Incomplete Data[J]. Computer Science. 2008,35(9)162-164.

12. Zhou Zhi-wen,HUANG Gao-ming,GAO Jun. Radar emitter identification algorithm based on deep learning[J].Journal of Xi dian University. 2017, 44(3). 77-82. 